\title{
La orejuela izquierda como fuente de accidentes cerebrovasculares: cerrar (y cómo), o no cerrar (y por qué)
}

\author{
Left atrial appendage as a source of stroke: to close (and how), \\ or not to close (and why)
}

\author{
R. Hernández-Estefanía ${ }^{1}$, B. Levy ${ }^{1}$, G. Rábago ${ }^{1}$
}

\section{RESUMEN}

La orejuela izquierda se considera la principal fuente de émbolos en los accidentes cerebrovasculares que se producen en pacientes con fibrilación auricular. El tratamiento con anticoagulantes orales reduce significativamente el riesgo de accidentes embólicos cerebrales en relación con la aspirina, pero se asocia a riesgo de complicaciones hemorrágicas, por lo que no siempre son utilizados. Se ha descrito que el cierre de la orejuela izquierda reduce la tasa de accidentes tromboembólicos y en la actualidad se recomienda en pacientes en fibrilación auricular sometidos a cirugía mitral, pero la formación de émbolos en estos pacientes puede deberse a otras causas, y la implicación de la orejuela podría ser menor de la que se presupone. Además, no todos los pacientes son candidatos para la anticoagulación oral, y no todos se mantienen en un rango terapéutico adecuado, lo que podría justificar la formación de trombos auriculares.

Existen diversos métodos para el cierre de la orejuela: sutura directa, exclusión epicárdica mediante grapadora o clip, u oclusión mediante los recientes dispositivos endovasculares de aplicación percutánea. Pero, según la bibliografía, la efectividad de estos métodos para ocluir completamente la orejuela, así como su seguridad y eficacia para la prevención de accidentes embólicos cerebrales, pueden presentar resultados dispares. Para aumentar la controversia, algunos métodos de cierre con sutura no revelan un claro beneficio e, incluso, en algunos se describe un aumento del riesgo de tromboembolismo.

Se presenta una revisión sobre la necesidad de cierre de la orejuela izquierda para la prevención de accidentes vasculares cerebrales, así como los diversos procedimientos descritos.

Palabras clave. Fibrilación auricular. Oclusión. Orejuela izquierda. Accidente cerebrovascular. Aanticoagulantes. \begin{abstract}
Left atrial appendage is considered the main source of emboli in stroke for patients with atrial fibrillation. Oral anticoagulant therapy significantly reduces the risk of cerebral embolic events as compared with aspirin, but it is associated with bleeding complications, and it is not considered an standard of care. Closure of the left atrial appendage reduces the rate of thromboembolic events, and currently it is recommended in patients with atrial fibrillation submitted to mitral valve surgery. However the formation of emboli in those patients may be due to other causes and the roll of the atrial appendage currently has not very well defined. Moreover, neither all patients are candidates for oral anticoagulation, nor patients with oral anticoagulant maintain a adequate therapeutic range, which could be justify the formation of atrial thrombi.
\end{abstract}

There are several methods to perform the closure of the appendage: endocavitary suture in concomitant mitral surgery, epicardial exclusion by stapling or clips, or endovascular occlusion by percutaneous devices. However the results seem inconclusive in regards of their effectiveness for complete occlusion of the appendage, safety, and efficacy to prevent cerebral embolic events. To increase the confusion, some authors reveal no clear benefit in suture closure, and even more others described an increased risk of thromboembolism.

We present a review of the left atrial appendage closure for prevention of stroke and the different procedures as previously described.

Key words. Atrial fibrillation. Occlusion. Left atrial appendage. Stroke. Anticoagulants.

\section{Servicio de Cirugía Cardiaca. Clínica Universi- dad de Navarra. Pamplona.}

Recepción: 26 de agosto de 2011

Aceptación provisional: 19 de diciembre de 2011

Aceptación definitiva: 10 de enero de 2012

\author{
Correspondencia: \\ Servicio de Cirugía Cardiovascular \\ Clínica Universidad de Navarra \\ Avda. Pío XII, 36 \\ 31008-Pamplona \\ E-mail: rhestefania@unav.es
}




\section{INTRODUCCIÓN}

La fibrilación auricular (FA) es la arritmia mantenida más frecuente en nuestros días $^{1}$, y se asocia a accidentes cerebrovasculares agudos (ACVA) de manera que al año, hasta un 5\% de pacientes en FA sin valvulopatía reumática concomitante presentan accidentes cerebrales isquémicos ${ }^{2}$.

Es bien conocida la implicación de la orejuela izquierda (OI) como fuente de émbolos cardiacos a la que se le ha llegado a considerar como: "el más letal de nuestros apéndices"3. Se sabe también que los ACVA de origen cardioembólico se asocian a un peor pronóstico a largo plazo ${ }^{4}$. En FA no asociada a valvulopatía reumática se calcula que más del $90 \%$ de pacientes presentan trombos en la $\mathrm{OI}^{5,6}$, y hasta el $57 \%$ en aquellos con valvulopatía reumática ${ }^{5}$. En pacientes con FA que no toman anticoagulantes orales (AO) un $15 \%$ presentan trombos en la aurícula izquierda ( $\mathrm{Al}$ ) (diagnosticados a través de ecocardiograma transesofágico -ETE-), localizándose en la OI en el 97\%?. Además, fisiopatológicamente, la ausencia de contracción de la OI facilita su dilatación, la ectasia de sangre y la formación del trombo ${ }^{8}$.

Pero, a pesar de todas estas evidencias, la formación de émbolos en pacientes con FA puede deberse a la existencia de otras fuentes con propiedades trombogénicas como la persistencia del foramen oval, o la arteriosclerosis $^{9-11}$, por lo que se ha llegado a considerar que la importancia atribuida a la OI como causante de la formación de émbolos podría ser bien menor ${ }^{8}$. Además, se ha calculado que en pacientes con FA asintomática y sin accidentes embólicos cerebrales previos, la prevalencia de trombos en la OI ronda sólo el 2,5\%, aunque los pacientes estudiados eran jóvenes, con FA transitoria y sin patología valvular concomitante ${ }^{12}$.

Los AO empleados en la prevención de ACVA de causa cardíaca, aunque hayan demostrado eficacia en comparación con los antiagregantes ${ }^{13}$, aumentan al doble la probabilidad de producir hemorragias cerebrales ${ }^{14}$. Por este motivo, no todos los pacientes con FA son considerados candidatos para el tratamiento con $\mathrm{AO}^{15}$, de ma- nera que se ha calculado que menos de la mitad de pacientes con FA se encuentran en tratamiento con $\mathrm{AO}$ en los tres meses después del alta hospitalaria ${ }^{16}$. Además se ha comprobado una alta variabilidad en el régimen de tratamiento dependiendo de cada institución ${ }^{17}$ y una dificultad para el mantenimiento de niveles terapéuticos adecuados dentro de un rango bastante estrecho ${ }^{18}$.

No existiendo un tratamiento médico definitivo, el cierre de la OI se recomienda en las guías europeas de actuación clínica para pacientes en FA sometidos a cirugía de la válvula mitral ${ }^{19}$, en donde algunos trabajos han descrito la reducción de la tasa de accidentes tromboembolicos cuando se asocia a cirugía cardiaca ${ }^{3,20-23}$. Sin embargo, en otros estudios no se revela este claro beneficio $^{24-26} \mathrm{o}$, incluso, se ha descrito que la eliminación de la OI podría perjudicar la respuesta hemodinámica al volumen y a la presión ${ }^{27}$.

Recientemente se ha descrito que las técnicas clásicas de cierre quirúrgico parecen poco eficaces en la obtención de una oclusión completa de la $\mathrm{OI}^{21}$, consecuencia tal vez de una marcada heterogenicidad en los diferentes tipos de técnicas de sutura. Por ese motivo, y para disminuir la agresión quirúrgica, se diseñaron los actuales tapones endocavitarios y clips de cierre externo -los cuales no están exentos de complicaciones derivadas del procedimiento ${ }^{28}$-, de los que se desconoce su eficacia real para ocluir totalmente la $\mathrm{OI}^{29}$.

En definitiva, existen dudas sobre la utilidad del cierre de la OI en la prevención de émbolos cerebrales. Tampoco existe un consenso en relación con la técnica o el dispositivo más efectivo, ni el tipo de pacientes en los que se debe aplicar.

\section{CIERRE QUIRÚRGICO DE LA OREJUELA IZQUIERDA}

Desde la primera vez que fue descrita hace más de sesenta $\operatorname{años}^{30}$, diversas técnicas quirúrgicas han sido utilizadas para el cierre de la OI (sutura simple, doble o circular), tanto por dentro de la AI como por fuera, pero siempre con un denominador 
común: el recelo por parte del cirujano a la manipulación de un tejido frágil que fácilmente podría complicar una cirugía valvular rutinaria ${ }^{20}$.

El desarrollo de grapadoras automáticas que realizan sutura y corte ha permitido un abordaje diferente, de manera que la OI se puede ocluir y/o seccionar, sin necesidad de abrir la AI. Siguiendo esta misma línea de abordaje epicárdico, se han diseñado dispositivos a modo de lazos corredizos que permiten estrangular la base de la OI hasta su oclusión ${ }^{31}$, así como clips epicardicos estériles de cierre automático como el Left atrial appendage clip ${ }^{\circledR}$ (AtriCure Inc.), que permite el cierre de la OI mediante abordajes mínimamente invasivos ${ }^{32}$. De muy reciente aparición, el dispositivo LARIAT® (SentreHEART) consiste en la implantación de un lazo introducido a través de una punción en el epicardio dirigido hacia la base de la OI mediante un imán endovascular introducido a través del septo interauricular (SIA) ${ }^{33}$.

\section{CIERRE ENDOVASCULAR DE LA OREJUELA IZQUIERDA}

La aparición de los recientes tapones endovasculares han permitido un nuevo abordaje de la OI, a través de la vena femoral y posterior punción del septo interauricular (SIA), siempre con apoyo de angiografía y ETE ${ }^{34}$.

Se han utilizado hasta el momento cuatro dispositivos ${ }^{29,35-37}$, en los que se ha observado una reducción de los ACVA, pero en los que se han observado complicaciones relacionadas con el procedimiento, así como algunos pacientes en los que no se alcanza la impermeabilidad total (Tabla 1).

Tabla 1. Principales dispositivos de oclusión percutánea de la orejuela izquierda, complicaciones y resultados

\begin{tabular}{|c|c|c|c|}
\hline & $\begin{array}{c}\text { PLAATO } \\
(\text { Ostermayer y col })^{35}\end{array}$ & $\begin{array}{l}\text { WATCHMAN } \\
\text { (Reddy y col) }^{28}\end{array}$ & $\begin{array}{c}\text { ACP } \\
(\text { Park y col })^{29}\end{array}$ \\
\hline CHAD2 (media $\pm \mathrm{DE})$ & $2,5 \pm 1,3$ & $2,2 \pm 1,2$ & ND \\
\hline $\mathrm{n}$ & 108 & 542 & 143 \\
\hline Sufusión pericárdica n (\%) & $2(1,8)$ & $27(5)$ & $4(3)$ \\
\hline Drenaje pericárdico $n(\%)$ & $3(2,7)$ & $22(4,8)$ & $5(4)$ \\
\hline Drenaje quirúrgico $n(\%)$ & $1(0,9)$ & $7(1,2)$ & - \\
\hline Embolización del dispositivo $n(\%)$ & ND & $3(0,6)$ & $2(1,3)$ \\
\hline Resultado ecográfico (ETE) & $13 \%$ fuga ligera & $\begin{array}{c}14 \% \text { flujo a través del } \\
\text { dispositivo }>5 \mathrm{~mm}\end{array}$ & ND \\
\hline Seguimiento meses & 9,8 & 18 & ND \\
\hline$A C V A \cap(\%)$ & $2(1,8)$ & $5(0,9)$ & $3(2)$ \\
\hline $\operatorname{AIT} n(\%)$ & $3(2,7)$ & 0 & 0 \\
\hline Conclusiones & Reducción riesgo ACVA $65 \%$ & Eficacia no inferior a los AO & $\begin{array}{l}\text { Seguro y } \\
\text { reproducible }\end{array}$ \\
\hline
\end{tabular}

AO: Anticoagulantes Orales; ACP: Amplatzer Cardiac Plug; ACVA: Accidente Cerebrovascular Agudo; AIT: Ataque Isquémico Transitorio; ETE: Ecocardiograma transesofágico, ND: No disponible. 
Se han publicado recientemente los resultados del estudio PROTECT $\mathrm{AF}^{37}$, en el que pacientes con FA de etiología no valvular se dividieron de manera aleatorizada en dos grupos: implantación del dispositivo WATCHMAN® (Atritech Inc.) mediante abordaje percutáneo, o tratamiento con AO. Al final del mismo se observó que el resultado en la prevención de nuevos ACVAS era de no inferioridad en relación con los pacientes tratados con AO. Aunque se observaban un alto porcentaje de eventos adversos, que incluían derrame pericárdico con necesidad de pericardiocentesis y/o drenaje quirúrgico $(4,8 \%)$, a los seis meses el flujo residual de sangre alrededor del dispositivo se había reducido a la mitad, debido al sellado tardío del mismo. Muy recientemente se han publicado los resultados de la continuación de este estudio con nuevos pacientes no aleatorizados ${ }^{28}$ (Tabla 1), en el que se observa un descenso de las complicaciones atribuido a la mejora en la curva de aprendizaje.

En la actualidad, el implante de dispositivos percutáneos se considera en la gran mayoría de los casos, en pacientes con contraindicación formal para la toma de AO.

\section{EFICACIA DE LAS TÉCNICAS QUIRÚRGICAS Y LOS DISPOSITIVOS EN LA OCLUSIÓN DE LA OREJUELA IZQUIERDA}

El principal inconveniente del cierre quirúrgico de la $\mathrm{OI}$ es el alto porcentaje de apéndices que quedan con un alto potencial embolígeno por no presentar impermeabilidad después del cierre ${ }^{38-39}$. En el trabajo publicado por Kanderian y col $^{21}$, se establecieron tres criterios para definir la ineficacia del cierre de la OI: a) OI permeable, b) exclusión de OI, pero apreciándose flujo persistente dentro del mismo y c) existencia de un fondo de saco residual en la OI después del cierre que es superior a $1 \mathrm{~cm}$. Aplicando estos criterios en una población de 137 pacientes a los que se les había excluido la OI mediante diferentes técnicas, los autores observaron hasta un $60 \%$ de pacientes que presentaban oclusión incompleta de OI. Dependiendo de la técnica utilizada, los porcentajes variaban; las orejuelas cerradas mediante sutura permanecían permeables o con fondo de saco residual en el $77 \%$ de los casos, a diferencia de las orejuelas amputadas (mediante sutura o grapadora y posterior excisión), en las que sólo el $27 \%$ estaban mal ocluidas. Estos resultados han sido corroborados por otros autores en estudios aleatorizados realizados en pacientes sometidos a cirugía coronaria, en los que el cierre se realizaba por fuera mediante sutura ${ }^{20}$ (Tabla 2 ).

La sutura de la orejuela por dentro de la AI durante la cirugía mitral no es tan sencilla como puede parecer en un primer momento, debido a una discordancia anatómica existente entre el ostium de la OI y el plano que forma la pared lateral auricular. Además, los bordes del orificio de entrada no están perfectamente delimitados en su parte más anterior e inferior, circunstancia que plantea dudas al cirujano de "por dónde" y "a qué profundidad" debe pasar los puntos, especialmente ante la proximidad de la arteria circunfleja (CX) (Fig. 1). A esta situación se debe añadir la dificultad anatómica que plantea una $\mathrm{AI}$ abierta y exangüe, lo que se puede traducir en puntos poco efectivos que pueden desgarrar el tejido cuando el corazón lata nuevamente ${ }^{23}$. Diversas técnicas de sutura se han utilizado $\left(\right.$ simple $^{40,41}$, doble $^{23}$, circular $\left.^{22}\right)$, con pobres resultados (Tabla 2), a excepción de una de las series publicadas ${ }^{22}$, en la que se obtiene casi un $90 \%$ de éxito mediante doble sutura (circular y simple), aunque los autores no analizan la presencia o no de fondo de saco residual. 
Tabla 2. Principales técnicas de oclusión quirúrgica de la orejuela izquierda

\begin{tabular}{|c|c|c|c|c|c|c|}
\hline Referencia & $\begin{array}{l}\text { Healey } \\
\mathrm{y} \mathrm{col}^{20}\end{array}$ & $\begin{array}{c}\text { Kanderian } \\
\mathrm{y} \mathrm{col}^{21}\end{array}$ & $\begin{array}{c}\text { García- } \\
\text { Fernández } \\
\text { y col }{ }^{22}\end{array}$ & $\begin{array}{c}\text { Katz } \\
\text { y col }^{23}\end{array}$ & $\begin{array}{c}\text { Salzberg } \\
\text { y col }^{32}\end{array}$ & $\begin{array}{c}\text { Blackshear } \\
\text { y col }^{31}\end{array}$ \\
\hline$n$ & 52 & 137 & 58 & 50 & 34 & 15 \\
\hline $\begin{array}{l}\text { Cirugía } \\
\text { concomitante }\end{array}$ & Coronaria & $\begin{array}{l}\text { Cardiaca } \\
\text { electiva }\end{array}$ & Valvular & Valvular & $\begin{array}{l}\text { Cardiaca } \\
\text { electiva }\end{array}$ & Ninguna \\
\hline Vía Abordaje & Esternotomía & Esternotomía & Esternotomía & Esternotomía & Esternotomía & Toracotomía \\
\hline \multicolumn{7}{|c|}{ Método de oclusión } \\
\hline - Aplicación & epicárdica & ND & endocavitaria & endocavitaria & epicárdica & epicárdica \\
\hline - Sutura n (\%) & $16(30,7)$ & $73(53,2)$ & $58(100)$ & $50(100)$ & - & - \\
\hline - Tipo sutura & $\begin{array}{c}\text { Simple } \\
\text { (circular o } \\
\text { continua) } \\
\end{array}$ & (ND) & $\begin{array}{c}\text { Doble } \\
\text { (circular y } \\
\text { continua) }\end{array}$ & $\begin{array}{c}\text { Doble } \\
\text { (continua) }\end{array}$ & - & - \\
\hline - Grapadora n (\%) & $36(69,2)^{(\mathrm{a})}$ & $12(8,7 \%)$ & - & - & - & - \\
\hline - Excisión ${ }^{(b)} n(\%)$ & - & $52(38 \%)$ & - & - & - & - \\
\hline- Otros & - & - & - & - & AED Clip & $\begin{array}{l}\text { Endoloop } \\
\text { snare }\end{array}$ \\
\hline \begin{tabular}{|l|}
$\begin{array}{l}\text { Seguimiento } \\
\text { (meses) }\end{array}$ \\
\end{tabular} & (2) & $\begin{array}{c}(8,1 \pm 12) \\
14 \text { días-9años } \\
\end{array}$ & $\begin{array}{c}\left(69,4^{(\mathrm{c})}\right) \\
1-329 \text { meses }\end{array}$ & \begin{tabular}{|c|}
$(64)$ \\
6 días-13 años \\
\end{tabular} & (3) & $(42 \pm 14)$ \\
\hline \multicolumn{7}{|c|}{ Evaluación de oclusión completa } \\
\hline -Método & ETE & ETE & ETE & ETE & $\mathrm{TAC}$ & ETE \\
\hline $\begin{array}{l}\text { - Criterio de } \\
\text { permeabilidad }\end{array}$ & $\begin{array}{l}\text { - OI residual } \\
(>1 \mathrm{~cm}) \\
\text { - Flujo } \\
\text { persistente }\end{array}$ & \begin{tabular}{|l|} 
- OI residual \\
$(>1 \mathrm{~cm})$ \\
- Flujo \\
persistente \\
- OI Permeable \\
\end{tabular} & $\begin{array}{l}\text { - Flujo entre } \\
\text { OI y AI }\end{array}$ & $\begin{array}{l}\text { - Flujo entre } \\
\text { OI y AI }\end{array}$ & $\begin{array}{l}\text { - Perfusión } \\
\text { en OI }\end{array}$ & $\begin{array}{l}\text { - Según } \\
\text { imagen } \\
\text { ecográfica }\end{array}$ \\
\hline \multicolumn{7}{|c|}{ Oclusión incompleta (\%) } \\
\hline - Sutura & 55 & 77 & 10,3 & 36 & - & - \\
\hline - Grapadora & $28^{(\mathrm{a})}$ & 100 & - & - & - & - \\
\hline -Excisión ${ }^{(b)}$ & - & 27 & - & - & - & - \\
\hline - Otras & - & - & - & 5,5 & 0 & ND \\
\hline Total & 34 & 60 & 10,3 & 36 & $0^{(\mathrm{d})}$ & ND \\
\hline \multicolumn{7}{|c|}{ Eventos embólicos n (\%): } \\
\hline$-A C V A$ & $1(1,9)$ & ND & ND & $1(5,5)^{(\mathrm{e})}$ & 0 & $2(14,2)$ \\
\hline$-A I T$ & $1(1,9)$ & ND & ND & $1(5,5)^{(\mathrm{e})}$ & 0 & 0 \\
\hline$-A m b o s$ & $2(3,8)$ & $18(13)$ & ND & $2(11,1)^{(\mathrm{e})}$ & 0 & $2(14,2)$ \\
\hline \multicolumn{7}{|c|}{ Complicaciones } \\
\hline $\begin{array}{l}\text { Relacionadas con el } \\
\text { procedimiento n (\%) }\end{array}$ & $9(17,3)$ & ND & ND & ND & 0 & $1(6,6)$ \\
\hline Tipo & $\begin{array}{c}\text { Rasgadura } \\
\text { de OI por } \\
\text { manipulación }\end{array}$ & ND & ND & ND & 0 & $\begin{array}{c}\text { - Toracotomia } \\
\text { urgente } \\
\text { (sangrado OI) }\end{array}$ \\
\hline
\end{tabular}

AED: Atrial exclusion device; ETE: Ecocardiograma transesofágico; OI: Orejuela izquierda; ND: no disponible;

TAC: Tomografía axial computarizada.

a) En algunos pacientes también se les fue realizada sutura; b) mediante tijera y sutura o mediante grapadora automática;

c) mediana; d) 2 pacientes no valorados y 2 pacientes mediante ETE; e) Datos en 18 pacientes con cierre incompleto de OI. 


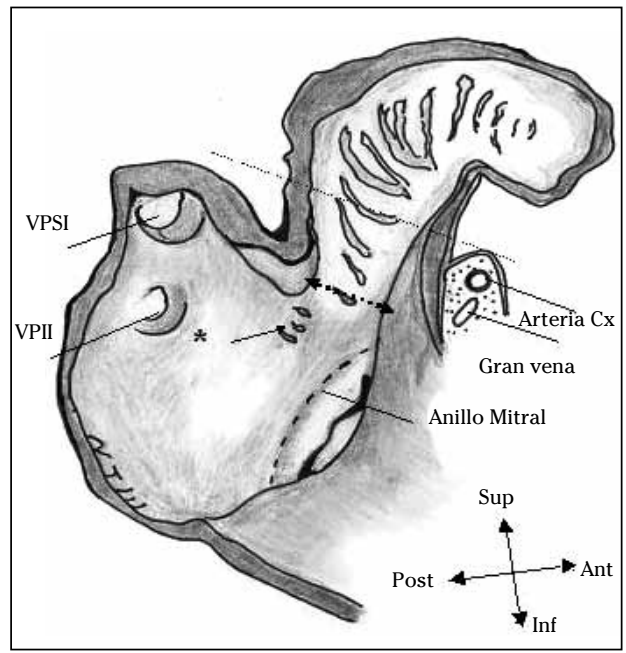

Cx: circunfleja; VPII: vena pulmonar inferior izquierda; VPSI: vena pulmonar superior izquierda.

Figura 1. Representación parcial de la cara lateral de la aurícula izquierda basado en estudios anatómicos previos ${ }^{42-50}$. La línea gruesa muestra el plano del ostium de la orejuela. La línea fina muestra el plano donde se aplican los dispositivos de cierre epicárdicos. El asterisco indica las zonas de adelgazamiento de la pared lateral, cerca del ostium de la orejuela ${ }^{50}$.

Las suturas realizadas del lado epicárdico parecen tener mejores resultados que las realizadas por dentro, aunque con un alto porcentaje de pacientes en los que se observa un fondo de saco residual que varía dependiendo de la técnica empleada: $58 \%$ cuando se utiliza la grapadora automática y $27 \%$ cuando se realiza excisión $^{21}$. Este alto porcentaje de orejuelas residuales con potencial de formación de trombos parece ser evidente si se tiene en cuenta la anatomía de la base de la OI (Fig. 1), ya que las grapadoras automáticas se colocan en realidad en un plano diferente al del ostium de la orejuela, lo que favorece que quede un fondo de saco residual. De la misma manera, la exclusión mediante suturas circulares realizadas en el lado epicárdico ocluyen a modo de esfínter (lo que puede favorecer que quede un orificio central permeable ${ }^{21}$ ), y los puntos circulares distribuidos se encuentran en planos diferentes (como en el caso de las grapadoras), en parte, por miedo de afectar las estructuras adyacentes.
Los actuales dispositivos endovasculares pueden generar dudas de su eficacia, debido a su diseño en relación con la anatomía de la OI, ya que son circulares para la oclusión de una superficie oval ${ }^{21,42}$ (lo que podría condicionar un sellado incompleto del orificio ${ }^{34}$ ), y afectar a la arteria CX por un exceso de estrés mecánico en la zona ${ }^{34}$. Además, muy cerca del ostium de la OI existen pequeñas hendiduras en las que la pared de la AI se adelgaza en comparación con el espesor del resto de la aurícula (Fig. 1), que podrían ser dañados por los catéteres empleados para la colocación de los dispositivos $^{42}$, y que justificarían las sufusiones pericardicas descritas con el procedimiento ${ }^{28,29,35}$ (Tabla 1).

En los resultados publicados para los dispositivos endovasculares, no se aplicaron los mismos criterios de impermeabilidad, en los que, dependiendo del dispositivo, un $13 \%$ presentan fuga ligera ${ }^{35}$ y un $14 \%$ flujo a través del dispositivo superior a 5 $\mathrm{mm}^{28}$, respectivamente.

Como se ha sugerido previamente, la existencia de flujo residual alrededor del dispositivo no excluye la formación de trombos $^{43}$, y la geometría de la OI (una estructura casi tubular pero de base ovalada), puede dificultar la evaluación de las fugas peri-dispositivo ${ }^{44}$.

Los recientes clips de oclusión epicárdica $^{32}$, así como los dispositivos que ocluyen mediante lazos o bandas, se implantan en un plano diferente al del ostium, circunstancia que pone en causa su eficacia futura. Además, los lazos (y las futuras bandas en fase todavía experimental), pueden presentar un defecto conceptual en su diseño, ya que ocluyen una superficie ovalada mediante una presión circular (lo que somete a una mayor tensión los bordes del diámetro mayor), favoreciendo los desgarros. Tal vez el que el procedimiento se realice en quirófano, presenta la ventaja de una posible reconversión a cirugía en caso de necesidad.

En general, la excisión completa presenta "a priori" mejores resultados de oclusión completa $^{21}$, pero se ha sugerido que podría comprometer la regulación volumétrica produciendo cierto grado de insuficiencia 
cardiaca $^{45}$. En cambio, la ligadura de la OI, con peores resultados de oclusión comple$\mathrm{ta}^{21}$, deja el apéndice intacto por lo que no comprometería la función cardiaca ${ }^{20}$. Por otro lado, el tamaño de la AI no parece influir en el resultado de permeabilidad ${ }^{20}$, aunque es discutible en cierres mediante suturas. Aurículas dilatadas de paredes finas no sólo dificultan la manipulación, como comprometen la sutura que debe idealmente mantener el diámetro longitudinal del óvalo. Para finalizar, se ha sugerido la posibilidad de que las propiedades endocrinas atribuidas a la OI puedan perjudicar el sellado de la OI por su secreción continua $^{38}$, aunque esto parece poco probable.

\section{UTILIDAD EN LA PREVENCIÓN DE FENÓMENOS EMBÓLICOS}

La verdadera utilidad del cierre de la OI para la prevención de fenómenos embólicos con origen cardiaco ${ }^{46}$, es un tema de discusión recurrente. Analizando todos los dispositivos y técnicas con estudios que presentan un seguimiento después del procedimiento, encontramos conclusiones muy dispares.

Algunos trabajos concluyen que el cierre de la OI no presenta ningún beneficio (Tabla 3a). En un estudio retrospectivo con 493 pacientes sometidos a exclusión de OI e implantación de prótesis (y con $\mathrm{AO}$ en el postoperatorio), el $67 \%$ de los pacientes que sufrieron un ACVA tenían la OI cerra$\mathrm{da}^{24}$. De la misma manera, en una serie de pacientes con cierre de OI y cirugía mitral asociada a cirugía coronaria, se describe una fuerte asociación entre fenómenos embólicos cerebrales y oclusión de la orejue$\mathrm{la}^{25}$. En otro trabajo se describe incluso, un aumento del riesgo de ACVA en pacientes con OI cuando no están bajo tratamiento con $\mathrm{AO}$, aunque este último estudio es unicéntrico, retrospectivo, y con pocos pacientes, lo que dificulta la extrapolación de los resultados ${ }^{26}$. No obstante, en ninguno de estos trabajos existe información en relación con el tipo de técnica utilizada para el cierre, ni de cual era el porcentaje de oclusión completa de las mismas ${ }^{24-26}$, en series con pacientes en los que no en todos se empleó $\mathrm{AO}^{25}$.

En contraposición, son mayores las evidencias a favor del beneficio del cierre de OI (Tabla 3 b), pero con resultados significativos en tan sólo un estudio en pacientes sometidos a cirugía mitral y oclusión de OI, donde se concluye que la ausencia del cierre de la orejuela es un predictor independiente de recurrencia de eventos embó$\operatorname{licos}^{22}$. En otros trabajos los resultados son menos consistentes, aunque se describe un menor porcentaje de eventos tromboembólicos en aquellos que tienen ocluida la $\mathrm{OI}^{20,21}$, y los ACVA registrados suceden en pacientes sin evidencia de trombos de origen cardiaco ${ }^{3}$. Pero en todos estos trabajos no se describe el régimen de tratamiento anticoagulante ${ }^{20} \mathrm{o}$ el número de pacientes con $\mathrm{AO}$ en el postoperatorio ${ }^{22}$. Tampoco hay información quirúrgica sobre el tipo de cierre ${ }^{3}$, o incluso, se describe un alto porcentaje de pacientes que presentan oclusión incompleta de $\mathrm{OI}^{21}$, por lo que los resultados no resultan muy concluyentes.

El estudio PROTECT AF realizado con el dispositivo WATCHMAN® (primer estudio aleatorizado realizado en pacientes con FA sin patología valvular concomitante), concluye que la implantación del dispositivo presenta una eficacia no inferior a los $\mathrm{AO}$, a pesar del mayor porcentaje de eventos adversos relacionados con el procedimiento. Si bien este trabajo parece decantar la balanza a favor de la oclusión de la OI, es necesario tener en cuenta que todos los pacientes a los que se les colocó el dispositivo mantuvieron AO durante 45 días después del procedimiento, y el $14 \%$ de ellos mantuvieron AO después de ese periodo ${ }^{37}$. No obstante, es la evidencia más sólida que se puede encontrar en la literatura, en relación con el beneficio del cierre de OI mediante dispositivos endovasculares. 
Tabla 3. Argumentos en contra y a favor del cierre de la orejuela izquierda para la prevención de accidentes embólicos en pacientes sometidos a cirugía cardiaca concomitante

\begin{tabular}{|c|c|c|c|c|c|c|c|}
\hline \multicolumn{8}{|c|}{ a) La oclusión de OI no presenta beneficio } \\
\hline & $\begin{array}{c}\text { Tipo } \\
\text { estudio }\end{array}$ & $\mathbf{n}$ & Cirugía & Evidencia & AO & A favor & En contra \\
\hline $\begin{array}{l}\text { Bando y } \\
\mathrm{col}^{24}\end{array}$ & $\mathrm{R}$ & 493 & $\begin{array}{l}\text { VMM } \\
\pm \text { Maze }\end{array}$ & $\begin{array}{l}\text { - } 67 \% \text { de pacientes } \\
\text { con ACVA tenían la OI } \\
\text { cerrada }\end{array}$ & \begin{tabular}{|l}
$-100 \%$ \\
de los \\
pacientes
\end{tabular} & $\begin{array}{l}\text { - El riesgo de ACVA no } \\
\text { disminuye con } \mathrm{AO} \text { ni } \\
\text { con cierre de } \mathrm{OI}\end{array}$ & $\begin{array}{l}\text { - Ausencia de } \\
\text { información sobre } \\
\text { el tipo de cierre de } \\
\text { Ol y el porcentaje } \\
\text { de oclusión } \\
\text { completa }\end{array}$ \\
\hline $\begin{array}{c}\text { Orszulak y } \\
\mathrm{col}^{25}\end{array}$ & $\mathrm{R}$ & 92 & $\begin{array}{c}\text { VMB } \\
\pm \mathrm{CC} \\
\pm \text { otros }\end{array}$ & $\begin{array}{l}\text { - Fuerte asociación } \\
\text { con ACVA en pacientes } \\
\text { sometidos a cirugía } \\
\text { mitral+CC y cierre de OI } \\
\text { (p>0,02) } \\
\text {-Cirugía mitral aislada y } \\
\text { cierre OI no aumenta el } \\
\text { riesgo de ACVA }\end{array}$ & $-\mathrm{ND}$ & $\begin{array}{l}\text { - FA previa u oclusión } \\
\text { de OI no se asocian al } \\
\text { riesgo de ACVA }\end{array}$ & $\begin{array}{l}\text { - Ausencia de } \\
\text { información sobre } \\
\text { el tipo de cierre } \\
\text { de OI y el \% de } \\
\text { oclusión completa } \\
\text { - No todos con AO }\end{array}$ \\
\hline $\begin{array}{l}\text { Almaha- } \\
\text { meed y } \\
\mathrm{col}^{26}\end{array}$ & $\mathrm{R}$ & 136 & Mitral & $\begin{array}{l}\text { - En pacientes con cierre } \\
\text { de OI, los ACVA son mas } \\
\text { frecuentes en pacientes } \\
\text { sin } A 0, \text { en comparación } \\
\text { con pacientes con AO } \\
(10 \% \text { frente a } 15 \%)\end{array}$ & $\begin{array}{l}-62 \% \text { de los } \\
\text { pacientes }\end{array}$ & $\begin{array}{l}\text { - Cierre de OI durante } \\
\text { cirugía mitral aumenta } \\
\text { el riesgo de fenómenos } \\
\text { tromboembólicos, } \\
\text { sobre todo en pacientes } \\
\text { sin AO }\end{array}$ & $\begin{array}{l}\text { - Información } \\
\text { sobre el tipo de } \\
\text { cierre de OI no } \\
\text { concluyente } \\
\text { - Ausencia de } \\
\text { información del } \\
\text { \% de oclusión } \\
\text { completa } \\
\end{array}$ \\
\hline \multicolumn{8}{|c|}{ b) La oclusión de OI es beneficiosa } \\
\hline & $\begin{array}{c}\text { Tipo } \\
\text { estudio }\end{array}$ & $\mathbf{n}$ & Cirugía & Evidencia & AO & A favor & En contra \\
\hline $\begin{array}{l}\text { Healey } \\
\mathrm{y} \mathrm{col}^{20}\end{array}$ & $\mathrm{P}, \mathrm{A}$ & 52 & $\mathrm{CC}$ & $\begin{array}{l}\text { - En pacientes con } \\
\text { cierre de OI menos } \\
\text { porcentaje de eventos } \\
\text { tromboembólicos }(2 \% \\
\text { frente a } 11 \%, \mathrm{p}=\mathrm{ns})\end{array}$ & $\begin{array}{l}\text { - Según el } \\
\text { criterio del } \\
\text { médico }\end{array}$ & \begin{tabular}{|l} 
- Cierre de OI es \\
reproducible, seguro y \\
no aumenta el tiempo \\
quirúrgico
\end{tabular} & $\begin{array}{l}\text { - Resultados no } \\
\text { significativos } \\
\text { - Ausencia de } \\
\text { homogeneicidad } \\
\text { del tratamiento } \\
\text { con AO } \\
\end{array}$ \\
\hline $\begin{array}{c}\text { García- } \\
\text { Fernández } \\
\mathrm{y} \mathrm{col}^{22}\end{array}$ & $\mathrm{R}$ & 58 & Mitral & $\begin{array}{l}\text { - En pacientes con cierre } \\
\text { de OI menos embolismo } \\
\text { sistémico (3,4\% frente a } \\
17 \%) \mathrm{p}=0,01\end{array}$ & \begin{tabular}{|l} 
- En \\
pacientes \\
con prótesis \\
mecánicas
\end{tabular} & $\begin{array}{l}\text { - Ausencia de cierre } \\
\text { de OI es un predictor } \\
\text { independiente de } \\
\text { recurrencia de eventos } \\
\text { embólicos }(\mathrm{p}=0,02)\end{array}$ & $\begin{array}{l}\text { - Hay pacientes } \\
\text { con AO en el } \\
\text { postoperatorio }\end{array}$ \\
\hline $\begin{array}{c}\text { Johnson } \\
\mathrm{y} \mathrm{col}^{3}\end{array}$ & $\mathrm{R}$ & 437 & ND & $\begin{array}{l}\text { - ACVA en el seguimiento } \\
\text { suceden en pacientes sin } \\
\text { evidencia de trombos en } \\
\text { OI o AI }\end{array}$ & $-\mathrm{ND}$ & $\begin{array}{l}\text { - Cierre profilactico de } \\
\text { OI es un método para } \\
\text { prevenir ACVA }\end{array}$ & $\begin{array}{l}\text { - Ausencia de } \\
\text { información sobre } \\
\text { el tipo de cierre } \\
\text { y de tratamiento } \\
\text { con AO }\end{array}$ \\
\hline $\begin{array}{c}\text { Katz } \\
\mathrm{y} \mathrm{col}^{23}\end{array}$ & $\mathrm{R}$ & 50 & Mitral & $\begin{array}{l}-4 \% \text { de ACVA (todos } \\
\text { en OI con cierre } \\
\text { incompleto) }\end{array}$ & $\begin{array}{l}\text { - ND (se } \\
\text { presupone) }\end{array}$ & $\begin{array}{l}\text { - Cierre incompleto } \\
\text { de OI es un potencial } \\
\text { mecanismo para } \\
\text { eventos embólicos }\end{array}$ & $\begin{array}{l}\text { - Hay pacientes } \\
\text { con AO en el } \\
\text { postoperatorio }\end{array}$ \\
\hline $\begin{array}{c}\text { Kanderian } \\
\mathrm{y} \mathrm{col}^{21}\end{array}$ & $\mathrm{R}$ & 137 & Mixta & $\begin{array}{l}\text { - En pacientes con } \\
\text { oclusión completa de OI, } \\
\text { menos ACVA/AIT (11\% } \\
\text { frente a } 15 \% \mathrm{p}=\mathrm{ns})\end{array}$ & $\begin{array}{l}-56 \% \text { con } \\
\text { AO }\end{array}$ & $\begin{array}{l}\text { - Tendencia al descenso } \\
\text { en la incidencia de ACVA/ } \\
\text { AIT en pacientes con } \\
\text { oclusión completa de OI }\end{array}$ & $\begin{array}{l}\text { - Alto porcentaje } \\
\text { de oclusión } \\
\text { incompleta de OI } \\
-46 \% \text { sin } \mathrm{AO} \\
\end{array}$ \\
\hline
\end{tabular}

A: aleatorizado; ACVA: Accidente cerebrovascular; AI: Aurícula izquierda; AIT: Ataque isquémico transitorio; CC: Cirugía coronaria; FA: Fibrilación auricular; N: número de orejuelas ocluidas; ND: No Disponible; ns: no significativo; P: Prospectivo; R: Retrospectivo; Maze: cirugía de la fibrilación auricular; VMB:Válvula mitral biológica; VMM: Válvula mitral mecánica. 


\section{CONSIDERACIONES FINALES}

En nuestra opinión, los argumentos científicos sobre el beneficio (o no) del cierre de la OI, se basan en estudios en los que se utilizan métodos de oclusión del OI muy dispares, realizados en pacientes con FA y otras patologías concomitantes, y con diferentes regímenes de $\mathrm{AO}$, lo que dificulta el análisis de los mismos. La eficacia y utilidad real del cierre de OI como prevención de ACVA sólo se demostrará en estudios de correcto diseño que permitan un seguimiento a largo plazo.

No obstante, existen notables evidencias anatómicas y clínicas a favor de la oclusión de la OI para la prevención de ACVA de origen cardiaco en pacientes con FA.

Además recientemente se ha sugerido que la orejuela es responsable del inicio de la FA hasta en el $27 \%$ de los pacientes ${ }^{47}$, por lo que parece justificado el cierre rutinario que actualmente se realiza en casi todos los centros quirúrgicos ${ }^{48,49}$.

En relación con los diferentes procedimientos de cierre no hay evidencias significativas que inclinen la balanza hacia uno u otro, lo que sugiere que la selección de la técnica debería ser escogida dependiendo del tipo de patología del paciente. De este modo, en pacientes con FA mantenidas y patología valvular concomitante susceptible de reparación o substitución quirúrgica, parece razonable el cierre de la OI a través de la AI durante el procedimiento mitral. Nuestro grupo recomienda la técnica de doble sutura después de eversión completa de la orejuela ${ }^{50}$, en la que se consigue fácilmente delimitar el plano del ostium de la OI, especialmente en su parte anterior e inferior, donde los bordes no están bien definidos $^{42,51}$.

En pacientes con FA sometidos a cirugía cardiaca en los que no se realiza la apertura de la AI, los clips de reciente aparición parecen ser una alternativa razonable, sobre todo en aquellos procedimientos en los que no existe la necesidad de circulación extracorpórea (CEC), aunque serian deseables mejoras en su diseño para evitar que se produzcan fondos de saco residuales potencialmente embolígenos. Las bandas y lazos en fase de desarrollo, pueden generar presión circular en una estructura ovalada, lo que podría condicionar complicaciones hemorrágicas graves, o dejar orejuelas con fondo de saco residual.

Los dispositivos endovasculares son muy prometedores, sobre todo en pacientes con FA, factores de riesgo de trombosis y contraindicaciones para AO que son sometidos a ablación percutánea, y podrían ser también aplicados en pacientes operados previamente, en los que las adherencias pericárdicas comprometerían otro tipo de abordaje. Resta, sin embargo conocer los resultados de permeabilidad a largo plazo, y la confirmación de la seguridad del procedimiento en centros que no dispongan de cirugía cardiaca.

Desde el punto de vista del cirujano acostumbrado a manipular la OI y "sufrir" su fragilidad (especialmente en AI dilatadas de paredes adelgazadas), un acceso mediante toracoscopia o con videoscopia mediante acceso con puertos, podría ser más seguro y de igual eficacia, en el tratamiento de la FA y cierre de la OI concomitante.

En definitiva, la oclusión de OI es un procedimiento que ha de ser considerado con la seriedad que merece: "una fuente de tromboembolismo cerebral de estructura frágil y acceso difícil, cuya oclusión completa puede resultar más compleja de lo que aparentemente se presupone".

\section{BIBLIOGRAFÍA}

1. Wolf PA, Аввотt RD, Kannel WB. Atrial fibrillation as an independent risk factor for stroke: the Framingham Study. Stroke 1991; 22: 983988.

2. Lloyd-Jones DM, Wang TJ, Leip EP, LaRson MG, LEVY D, VASAN RS et al. Lifetime risk for development of atrial fibrillation: the Framingham Heart Study. Circulation 2004; 110: 1042-1046.

3. Johnson WD, Ganjoo AK, Stone CD, SRIvyas RC, HowARD M. The left atrial appendage: our most lethal human attachment! Surgical implications. Eur J Cardiothorac Surg 2000; 17: 718-722. 
4. Henriksson KM, Farahmand B, Johansson S. Survival after stroke: the impact of CHADS2 score and atrial fibrillation. Int J Cardiol 2010; 141: 18-23.

5. Blackshear JL, Odell JA. Appendage obliteration to reduce stroke in cardiac surgical patients with atrial fibrillation. Ann Thorac Surg 1996; 61: 755-759.

6. MANNING WJ. Atrial fibrillation, transesophageal echo, electrical cardioversion, and anticoagulation. Clin Cardiol 1994; 17: 639-640.

7. Al-SAady NM, OBel OA, CAMm AJ. Left atrial appendage: structure, function, and role in thromboembolism. Heart 1999; 82: 547-554.

8. Whitlock RP, Healey JS, ConNolly SJ. Left atrial appendage occlusion does not eliminate the need for warfarin. Circulation 2009; 120 : 1927-1932.

9. Okura H, Inoue H, Tomon M, Nishiyama S, YosHIKAWA T, Yoshida K. Is the left atrium the only embolic source in ischemic stroke patients with nonvalvular atrial fibrillation? Am J Cardiol 1999; 84: 1259-1261.

10. Hart RG, Pearce LA, Miller VT, Anderson DC, Rothrock JF, Albers GW et al. Cardioembolic vs. noncardioembolic strokes in atrial fibrillation: frequency and effect of antithrombotic agents in the stroke prevention in atrial fibrillation studies. Cerebrovasc Dis 2000; 10: $39-43$

11. Blackshear JL, Pearce LA, Hart RG, Zabalgoitia M, Labovitz A, Asinger RW et al. Aortic plaque in atrial fibrillation: prevalence, predictors, and thromboembolic implications. Stroke 1999; 30: 834-840.

12. Stöllberger C, Chnupa P, Kronik G, Brainin M, Finsterer J, Schneider B et al. Transesophageal echocardiography to assess embolic risk in patients with atrial fibrillation. ELAT Study Group. Embolism in Left Atrial Thrombi. Ann Intern Med 1998; 128: 630-638.

13. ACTIVE Writing Group of the ACTIVE InVEstigators, Connolly S, Pogue J, Hart R, Pfeffer M, HohNloser S, Chrolavicius S et al. Clopidogrel plus aspirin versus oral anticoagulation for atrial fibrillation in the Atrial fibrillation Clopidogrel Trial with Irbesartan for prevention of Vascular Events (ACTIVE W): a randomised controlled trial. Lancet 2006; 367: 1903-1912.

14. Hart RG, Pearce LA, Aguilar Mi. Meta-analysis: antithrombotic therapy to prevent stroke in patients who have nonvalvular atrial fibrillation. Ann Intern Med 2007; 146: 857-867.

15. Reynolds MR, Shah J, Essebag V, Olshansky B, Friedman PA, Hadjis T et al. Patterns and pre- dictors of warfarin use in patients with newonset atrial fibrillation from the FRACTAL Registry. Am J Cardiol 2006; 97: 538-543.

16. Humphries KH, Jackevicius C, Gong Y, Svensen L, Cox J, Tu JV et al. Canadian Cardiovascular Outcomes Research Team. Population rates of hospitalization for atrial fibrillation/flutter in Canada. Can J Cardiol 2004; 20: 869-876.

17. Connelly SJ, Pogue J, Eikelboom J, Flaker G, ComMERFORD P, FRANZOSI MG et al. ACTIVE W Investigators. Benefit of oral anticoagulant over antiplatelet therapy in atrial fibrillation depends on the quality of international normalized ratio control achieved by centers and countries as measured by time in therapeutic range. Circulation 2008; 118: 2029-2037.

18. Holmes DR JR, Schwartz RS. Left atrial appendage occlusion eliminates the need for warfarin. Circulation 2009; 120: 1919-1926.

19. Fuster V, Ryden LE, Cannom DS, Crijns HJ, Curtis AB, Ellenbogen KA et al. ACC/AHA/ESC 2006 guidelines for the management of patients with atrial fibrillation-executive summary: a report of the American College of Cardiology/American Heart Association Task Force on Practice Guidelines and the European Society of Cardiology Committee for Practice Guidelines (Writing Committee to Revise the 2001 Guidelines for the Management of Patients with Atrial Fibrillation). Eur Heart J 2006; 27: 1979-1930.

20. Healey JS, Crystal E, lamy A, Teoh K, Semelhago L, Hohnloser SH et al. Left atrial appendage occlusion study (LAAOS): results of a randomized controlled pilot study of left atrial appendage occlusion during coronary bypass surgery in patients at risk for stroke. Am Heart J 2005; 150: 288-293.

21. Kanderian AS, Gillinov AM, Pettersson GB, Klein AL. Success of surgical left atrial appendage occlusion techniques assessed by transesophageal echocardiography. J Am Coll Cardiol 2008; 52: 924-929.

22. Garcia-Fernandez MA, Perez-David E, Quiles J, Peralta J, Garcia-Rojas I, Bermejo J et al. Role of left atrial appendage obliteration in stroke reduction in patients with mitral valve prosthesis: a transesophageal echocardiographic study. J Am Coll Cardiol 2003; 42: 1253-1258.

23. Katz ES, Tsiamtsiouris T, Applebaum RM, Schwartzbard A, Tunick PA, Kronzon I. Surgical left atrial appendage ligation is frequently incomplete: a transesophageal echocardiograhic study. J Am Coll Cardiol 2000; 36: 468471 . 
24. Bando K, Kobayashi J, Hirata M, Satoh T, Niwaya $\mathrm{K}$, TAguSARI $\mathrm{O}$ et al. Early and late stroke after mitral valve replacement with a mechanical prosthesis: risk factor analysis of a 24-year experience. J Thorac Cardiovasc Surg 2003; 126: 358-364.

25. Orszulak TA, Schaff HV, Pluth JR, Danielson GK, PugA FJ, ILSTRUP DM et al. The risk of stroke in the early postoperative period following mitral valve replacement. Eur J Cardiothorac Surg 1995; 9: 615-620.

26. Almahameed ST, Khan M, Zuzek RW, Juratli N, BELDEN WA, Asher CR et al. Left atrial appendage exclusion and the risk of thromboembolic events following mitral valve surgery. J Cardiovasc Electrophysiol 2007; 18: 364-366.

27. Stollberger C, Schneider B, Finsterer J. Elimination of the left atrial appendage to prevent stroke or embolism? Anatomic, physiologic, and pathophysiologic considerations. Chest 2003; 124: 2356-2362.

28. Reddy VY, Holmes D, Doshi SK, Neuzil P, Kar S. Safety of percutaneous left atrial appendage closure: results from the Watchman Left Atrial Appendage System for embolic protection in Patients with AF (PROTECT AF) Clinical Trial and the Continued Access Registry. Circulation 2011; 123: 417-424.

29. Park JW, Bethencourt A, Sievert H, Santoro G, Meier B, WALSh K et al. Left atrial appendage closure with amplatzer cardiac plug in atrial fibrillation: initial european experience. Catheter Cardiovasc Interv 2011; 77: 700-706.

30. MADDEN JL. Resection of the left auricular appendix; a prophylaxis for recurrent arterial emboli. J Am Med Assoc 1949; 140: 769-772.

31. Blackshear JL, Johnson WD, Odell JA, BaKer VS, HowARD M, PEARCE L et al.Thoracoscopic extracardiac obliteration of the left atrial appendage for stroke risk reduction in atrial fibrillation. J Am Coll Cardiol 2003; 42: 12491252.

32. Salzberg SP, Plass A, Emmert MY, Desbiolles L, AlKADHI H, GRÜNENFELdER J et al. Left atrial appendage clip occlusion: early clinical results. J Thorac Cardiovasc Surg 2010; 139:12691274.

33. Bartus K, Bednarek J, Myc J, Kapelak B, SAdowski J, LELAKOWSKI J. Feasibility of closed-chest ligation of the left atrial appendage in humans. Heart Rhythm 2011; 8: 188-193.

34. Cruz-Gonzalez I, YAN BP, Lam YY. Left atrial appendage exclusion: state-of-the-art. Catheter Cardiovasc Interv 2010; 75: 806-813.
35. Ostermayer SH, Reisman M, Kramer PH, Matthews RV, Gray WA, Block PC et al. Percutaneous left atrial appendage transcatheter occlusion (PLAATO system) to prevent stroke in high-risk patients with non-rheumatic atrial fibrillation: results from the international multi-center feasibility trials. J Am Coll Cardiol 2005; 46: 9-14.

36. ChIAm PT, RuIz CE. Percutaneous transcatheter left atrial appendage exclusion in atrial fibrillation. J Invasive Cardiol 2008; 20: 109113.

37. Holmes DR JR, Reddy VY, Turi Z, Doshi SK, SieVERT H, BuchBinder $\mathrm{M}$ et al, for the PROTECT AF Investigators. Percutaneous closure of the left atrial appendage versus warfarin therapy for prevention of stroke in patients with atrial fibrillation: a randomized non-inferiority trial. Lancet 2009; 374: 534-542.

38. Stöllberger C, Finsterer J, Schneider B. Arguments against left atrial appendage occlusion for stroke prevention. Stroke 2007; 38: e 77.

39. Rosenzweig BP, Katz E, Kort S, Schloss M, KronzON I. Thromboembolus from a ligated left atrial appendage. J Am Soc Echocardiogr 2001; 14: 396-398.

40. Schneider B, Stollberger C, Sievers HH. Surgical closure of the left atrial appendage: a beneficial procedure? Cardiology 2005; 104: 127 132.

41. Lynch M, Shanewise JS, Chang GL, Martin RP, Clements SD. Recanalization of the left atrial appendage demonstrated by transesophageal echocardiography. Ann Thorac Surg 1997; 63: 1774-1775.

42. Su P, McCarthy KP, Ho SY. Occluding the left atrial appendage: anatomical considerations. Heart 2008; 94: 1166-1170.

43. Schneider B, Finsterer J, Stöllberger C. Effects of percutaneous left atrial appendage transcatheter occlusion (PLAATO) on left atrial structure and function. J Am Coll Cardiol 2005; 45: 634-635.

44. Schwartzman D, Katz WE, Smith AJ, Anderson WD. Malpositioning of a left atrial appendage occlusion device? A case with implications for percutaneous transcatheter left atrial appendage occlusion device therapy. Heart Rhythm 2007; 4: 648-650.

45. Yoshihara F, Nishikimi T, Kosakai Y, Isobe F, MatSUOKA $\mathrm{H}$, TAKISHITA $\mathrm{S}$ et al. Atrial natriuretic peptide secretion and body fluid balance after bilateral atrial appendectomy by the 
maze procedure. J Thorac Cardiovasc Surg 1998; 116: 213-219.

46. Dawson AG, Asopa S, Dunning J. Should patients undergoing cardiac surgery with atrial fibrillation have left atrial appendage exclusion? Interact Cardiovasc Thorac Surg 2010; 10: 306-311.

47. Di Biase L, Burkhardt JD, Mohanty P, Sanchez J, Mohanty S, Horton R et al. Left atrial appendage: an underrecognized trigger site of atrial fibrillation. Circulation 2010; 122: 109-118.

48. Hernández-Estefanía R, Neves J, Abecasis M, Moradas Ferreira M, Ribeiras R, Cavaco D et al. Tratamiento quirúrgico sin circulación extracorpórea de la fibrilación auricular aislada para la prevención secundaria de accidentes cerebrovasculares isquémicos agudos. J Cir Cardiov 2006; 13: 163-170.

49. Gillinov AM. Advances in surgical treatment of atrial fibrillation. Stroke 2007; 38: (2 Suppl.) 618-623.

50. Hernandez-Estefania R, Praschker BL, BastaRRIKA G, RABAGO G. Left atrial appendage occlusion by invagination and double suture technique. Eur J Cardiothorac Surg 2011. doi:10.1016/j.ejcts.2011.05.022

51. Cabrera JA, Ho SY, Climent V, SÁnchez-Quintana D. The architecture of the left lateral atrial wall: a particular anatomic region with implications for ablation of atrial fibrillation. Eur Heart J 2008; 29: 356-362. 